\title{
An Input Error Method for Parameter Identification of a Class of Euler-Lagrange Systems
}

\author{
Adolfo Perrusquía \\ School of Aerospace, Transport \\ and Manufacturing \\ Cranfield University \\ Bedford, UK \\ Adolfo.Perrusquia-Guzman@ cranfield.ac.uk
}

\author{
Ruben Garrido \\ Departamento de Control Automático \\ CINVESTAV-IPN \\ Mexico city, Mexico \\ garrido@ctrl.cinvestav.mx
}

\author{
Wen $\mathrm{Yu}$ \\ Departamento de Control Automático \\ CINVESTAV-IPN \\ Mexico city, Mexico \\ yuw@ctrl.cinvestav.mx
}

\begin{abstract}
In this paper, an input error identification algorithm for a class of Euler-Lagrange systems is proposed. The algorithm has a state-observer structure which uses the input error between the real system and an estimated model instead of the output error. Both systems are controlled by two Proportional-Derivative (PD) controllers with the same gain values. An excitation signal is added to the PD controllers to guarantee parameter estimates convergence. Stability of the complete identification method and parameter estimates convergence are assessed via Lyapunov stability theory. Simulation studies are carried out to verify the approach.
\end{abstract}

\section{INTRODUCTION}

Parameter identification is a well known control problem which seeks to estimate the parameters of a system. These parameters gives useful qualitative information for tuning controllers gains for the design of model-based feedforward and feedback controllers [1].

There exist several kinds of methodologies to estimate system parameters either off-line or on-line. Some methods are well known algorithms such as least mean squares (LMS) [2] [3], recursive least squares (RLS) [4] and neural networks, which work in open-loop and require BIBO (bounded-input bounded-output) stability, which is not always the case. Other algorithms work in closed-loop such as adaptive controllers [5], [6] where the identification and the control law are executed simultaneously, e.g., the famous algorithm of Slotine \& Li [7]-[9] and sliding mode identification [10]-[12]. These adaptive controllers use the parameter estimates to compute the controller [13], [14] and exhibit good performance, but without a Persistency of Excitation (PE) condition [15] the parameter estimates would not converge to their true vales [16].

Most of the identification algorithms are off-line and do not give a convergence analysis proof [2]. In other words, it is argued that the identification algorithm is iterative and the parameter estimates will converge to values close to their real parameters values. However, there is no mathematical proof using either Lyapunov stability theory [17] or the Banach contraction property [18], [19] which are two of the most common mathematical tools for convergence analysis. Furthermore, if the identification algorithm does not consider the PE condition then the estimates will not converge to their real values.

On-line approaches have been considered by using a Kalman Filter [20] and a Kalman Active-Observer [21] to estimate unknown parameters of a system. However, the Kalman filter requires a careful tuning procedure. Another approach is the multi-estimator (ME) approach, which is a supervisory control that finds the best approximated model among other candidates. It performs well but it does not guarantee parameter convergence [4]. The closed-loop output error (CLOE) algorithm has been studied for the identification of discrete linear time systems. It provides unbiased estimates and the controller feeds the identification algorithm such as in the case of an indirect adaptive controller [22]-[24], but it requires values of the parameter estimates obtained previously under open loop conditions. New CLOE approaches [25] are developed by using control inputs instead of measurements of the generalized coordinates and their respective derivatives.

The closed-loop input error (CLIE) algorithm is proposed in [2], where an estimated model of a DC servomechanism is in closed-loop with a Proportional Derivative (PD) controller, and the input error between the real DC motor and its estimated model feeds an identification algorithm that subsequently update the estimate model. Its stability is proven theoretically without considering disturbances by means of Lyapunov theory, and experimentally by using a laboratory prototype. However, the PE condition [26] on parameter convergence is not analysed.

Motivated by the above comments, this work reports an online parameter identification algorithm using a Closed Loop Input Error (CLIE) approach for a class of Euler-Lagrange systems. Two identification cases are analysed: exact model matching and estimation error. The convergence of the parameter estimates are assessed using Lyapunov stability theory which takes into account the PD controller employed for stabilizing the Euler-Lagrange system, the PE signal, and the estimated model. 


\section{A Class of Euler-Lagrange Systems}

Consider the following dynamic equation of a class of EulerLagrange systems:

$$
\mathcal{J} \ddot{x}+\mathcal{R} \dot{x}+\mathcal{P} x=Q_{n c}+Q_{c}
$$

where $\mathcal{J} \in \mathbb{R}^{n \times n}$ is a symmetric inertia matrix, $\mathcal{R} \in \mathbb{R}^{n \times n}$ is a matrix with dissipative terms, $\mathcal{P} \in \mathbb{R}^{n \times n}$ is a compliance matrix, $Q_{n c} \in \mathbb{R}^{n}$ are the non-conservatives forces and $Q_{c} \in \mathbb{R}^{n}$ are the conservative forces, $x, \dot{x}, \ddot{x} \in \mathbb{R}^{n}$ are the generalized coordinates and their respective derivatives.

This class of Euler-Lagrange systems stands to many mechanical, electrical and hydraulic models, e.g., for mechanical and electrical systems: $\mathcal{J}$ represents the inertia or inductance, $\mathcal{R}$ can represents friction, a damper or a resistor, $\mathcal{P}$ represents a spring or a capacitance, $Q_{c}$ represents components due to the potential energy as gravitational terms, $Q_{n c}$ are the external inputs such as torques, forces, voltages or current sources. The following assumption is required

Assumption 1: The inertial matrix $\mathcal{J}$ is constant and positive definite with eigenvalues different to zero, i.e, $\mathcal{J}=\mathcal{J}^{\top}>0$. Now consider the following alternative form of the dynamics (1):

$$
\ddot{x}=-A \dot{x}-C x+B Q_{n c}+D
$$

where $A=\mathcal{J}^{-1} \mathcal{R}, C=\mathcal{J}^{-1} \mathcal{P}, B=\mathcal{J}^{-1}$, and $D=\mathcal{J}^{-1} Q_{c}$. This particular version is needed to apply the input error identification technique.

\section{Closed LOOP INPUT ERROR (CLIE) IDENTIFICATION TECHNIQUE}

The closed loop input error (CLIE) identification algorithm [2] is shown in Fig. 1. The diagram is composed of the EulerLagrange system in parallel with an estimated model. Both systems are controlled by a PD control law, which stabilize (2) without knowledge on its parameters, and tuned with the same gains [27]. The input error is used by an identification algorithm to update the estimates of the estimated model.

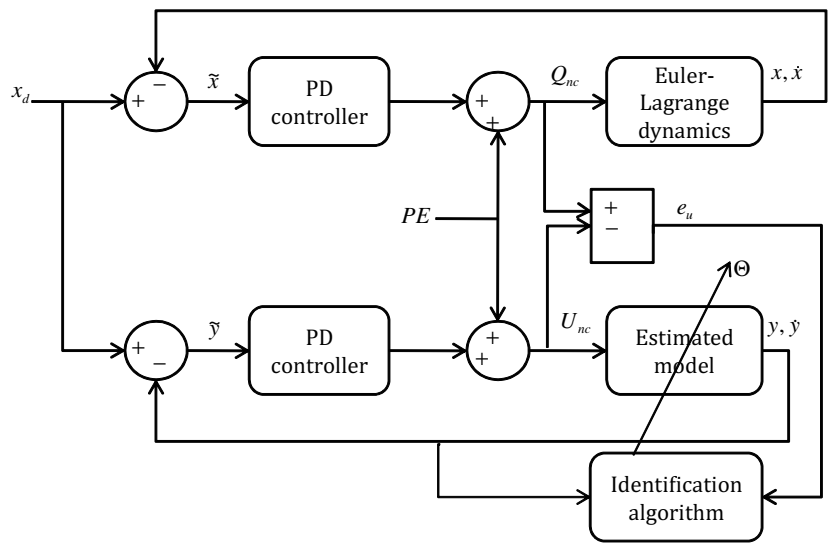

Fig. 1. Block diagram of the closed loop input identification technique

The next PD control law is applied to the Euler-Lagrange dynamics (2)

$$
Q_{n c}=K_{p} \widetilde{x}-K_{d} \dot{x}+P E,
$$

where $K_{p}=\operatorname{diag}\left(k_{p_{1}}, \cdots, k_{p_{n}}\right) \in \mathbb{R}^{n \times n}$ and $K_{d}=$ $\operatorname{diag}\left(k_{d_{1}}, \cdots, k_{d_{n}}\right) \in \mathbb{R}^{n \times n}$ denote the proportional and derivative gain matrices, respectively; where $k_{p_{i}}, k_{d_{i}}>0$, $i=1, \cdots n$. The error vector is defined as $\widetilde{x}=x_{d}-x$ where $x_{d} \in \mathbb{R}^{n}$ is a constant desired reference and $P E$ is a bounded excitation signal.

Remark 1: The control law $Q_{n c}$ is used only to stabilize the plant and does not guarantee convergence to the desired trajectory. Hence, the PD control law (3) is the simplest control law which can achieve this objective.

Remark 2: Proportional-Integral-Derivative (PID) is commonly used for system identification. However, the integral term could destabilize [28] the closed-loop dynamics of the estimated model.

The Euler-Lagrange dynamics (2) under the PD control law (3) yields the closed-loop dynamics

$$
\ddot{x}=-\left(A+B K_{d}\right) \dot{x}-C x+B\left(K_{p} \tilde{x}+P E\right)+D=-\Phi^{\top} \Theta
$$

where $\Phi=\Phi(\widetilde{x}, x, \dot{x}, P E) \in \mathbb{R}^{p \times n}$ is the regressor matrix and $\Theta \in \mathbb{R}^{p}$ is the unknown parameters vector which are defined as

$$
\Phi=\left[\begin{array}{c}
I \otimes \dot{x} \\
I \otimes x \\
-I \otimes Q_{n c} \\
-I
\end{array}\right], \quad \Theta=\left[\begin{array}{c}
\operatorname{vec}(A) \\
\operatorname{vec}(C) \\
\operatorname{vec}(B) \\
D
\end{array}\right]
$$

where $\otimes$ denotes the Kronecker product, $\operatorname{vec}(P) \in \mathbb{R}^{w v}$ denote the vectorization of matrix $P \in \mathbb{R}^{w \times v}$ and $I$ is the identity matrix of an appropriate dimension.

The estimated model has the following structure

$$
\ddot{y}=-\widehat{A} \dot{y}-\widehat{C} y+\widehat{B} U_{n c}+\widehat{D}
$$

where $\widehat{A}, \widehat{B}, \widehat{C}, \widehat{D}$ are estimates of $A, B, C$, and $D$, respectively, and $y \in \mathbb{R}^{n}$ is the state of the estimated model. The control law $U_{n c}$ has the same structure as in (3) as

$$
U_{n c}=K_{p} \widetilde{y}-K_{d} \dot{y}+P E
$$

with $\widetilde{y}=x_{d}-y$. The estimated model (6) under the PD control law (7) gives

$$
\ddot{y}=-\left(\widehat{A}+\widehat{B} K_{d}\right) \dot{y}-\widehat{C} y+\widehat{B}\left(K_{p} \widetilde{y}+P E\right)+\widehat{D}=-\Phi_{y}^{\top} \widehat{\Theta} .
$$

The term $\Phi_{y}=\Phi_{y}(\widetilde{y}, y, \dot{y}, P E) \in \mathbb{R}^{p \times n}$ is a regressor matrix and $\widehat{\Theta} \in \mathbb{R}^{p}$ is an estimate of the parameter vector $\Theta$ which are defined as

$$
\Phi_{y}=\left[\begin{array}{c}
I \otimes \dot{y} \\
I \otimes y \\
-I \otimes U_{n c} \\
-I
\end{array}\right], \quad \widehat{\Theta}=\left[\begin{array}{c}
\operatorname{vec}(\widehat{A}) \\
\operatorname{vec}(\widehat{C}) \\
\operatorname{vec}(\widehat{B}) \\
\widehat{D}
\end{array}\right] .
$$

\section{A. Exact matching}

Consider the case that the identification algorithm exactly estimates the parameters of the Euler-Lagrange system. Let define the output error as

$$
e=\widetilde{y}-\widetilde{x}=x-y
$$


The closed-loop error dynamics between the dynamics (2) and the estimated model (6) is

$$
\begin{aligned}
\ddot{e} & =-\bar{A} \dot{e}-\bar{B} e+\widetilde{A} \dot{y}+\widetilde{C} y-\widetilde{B} U_{n c}-\widetilde{D} \\
& =-\bar{A} \dot{e}-\bar{B} e+\Phi_{y}^{\top} \widetilde{\Theta}
\end{aligned}
$$

where $\bar{A}=A+B K_{d}, \bar{B}=C+B K_{p}, \widetilde{\Theta}=\widehat{\Theta}-\Theta \in \mathbb{R}^{p}$ is the parametric error vector. The parametric error is defined as

$$
\widetilde{\Theta}=\widehat{\Theta}-\Theta=\left[\begin{array}{c}
\operatorname{vec}(\widehat{A})-\operatorname{vec}(A) \\
\operatorname{vec}(\widehat{C})-\operatorname{vec}(C) \\
\operatorname{vec}(\widehat{B})-\operatorname{vec}(B) \\
\widehat{D}-D
\end{array}\right] .
$$

The input error $e_{u}$ is given by the difference between the PD control laws as

$$
\begin{aligned}
e_{u} & =U_{n c}-Q_{n c} \\
& =K_{p} e+K_{d} \dot{e}
\end{aligned}
$$

The next theorem establishes the stability and parameter convergence of the CLIE approach applied to the parameter estimation of any Euler-Lagrange system of the form (2).

Theorem 1: Consider the Euler-Lagrange system dynamics (2) in closed-loop with the PD control law (3), and the estimated model (6) in closed-loop with control law (7). If the parameter estimates $\widehat{\Theta}$ are updated as

$$
\dot{\hat{\Theta}}=-\Gamma \Phi_{y} K_{d} K_{p}^{-1} e_{u},
$$

where $\Gamma=\Gamma^{\top}>0 \in \mathbb{R}^{p \times p}$ is a diagonal matrix gain, and $K_{p}^{-1} K_{d} \bar{A}-I>0$ then $\widetilde{\Theta}, e, \dot{e}, y, \dot{y}$ and $\Phi_{y}$ remain bounded and the input error $e_{u}$ converges to zero.

Proof: Consider the following Lyapunov function

$$
\begin{aligned}
V= & \frac{1}{2} e_{u}^{\top} K_{p}^{-1} e_{u}+\frac{1}{2} \widetilde{\Theta}^{\top} \Gamma^{-1} \widetilde{\Theta} \\
& +\frac{1}{2} e^{\top}\left[\bar{B}^{\top} K_{d} K_{p}^{-1} K_{d}+K_{p}\left(K_{p}^{-1} K_{d} \bar{A}-I\right)\right] e
\end{aligned}
$$

This function is positive definite if $K_{p}^{-1} K_{d} \bar{A}-I>0$. The derivative of (15) with respect to the time is

$$
\begin{aligned}
\dot{V}= & e_{u}^{\top} K_{p}^{-1}\left(K_{p} \dot{e}+K_{d} \ddot{e}\right)+\widetilde{\Theta}^{\top} \Gamma^{-1} \dot{\widetilde{\Theta}} \\
& +e^{\top}\left(K_{d} \bar{A}+\bar{B}^{\top} K_{d} K_{p}^{-1} K_{d}-K_{p}\right) \dot{e} \\
= & -e^{\top} K_{d} \bar{B} e-\dot{e}^{\top} K_{d}\left(K_{p}^{-1} K_{d} \bar{A}-I\right) \dot{e} \\
& +\widetilde{\Theta}^{\top}\left(\Gamma^{-1} \dot{\widetilde{\Theta}}+\Phi_{y} K_{d} K_{p}^{-1} e_{u}\right)
\end{aligned}
$$

If the update law is chosen as (14), then the time-derivative of the Lyapunov function simplifies to

$$
\begin{aligned}
\dot{V} & =-e^{\top} K_{d} \bar{B} e-\dot{e}^{\top} K_{d}\left(K_{p}^{-1} K_{d} \bar{A}-I\right) \dot{e} \\
& =-\left[\begin{array}{c}
e \\
\dot{e}
\end{array}\right]^{\top} \underbrace{\left[\begin{array}{cc}
K_{d} \bar{B} & 0 \\
0 & K_{d}\left(K_{p}^{-1} K_{d} \bar{A}-I\right)
\end{array}\right]}_{Q \in \mathbb{R}^{2 n \times 2 n}}\left[\begin{array}{c}
e \\
\dot{e}
\end{array}\right] \\
& \leq-\lambda_{\min }(Q)\|E\|^{2}
\end{aligned}
$$

where $E=\left[e^{\top}, \dot{e}^{\top}\right]^{\top}$ and $\lambda_{\min }(Q)$ is the minimum eigenvalue of matrix $Q$. The matrix $Q$ is positive definite if
$K_{p}^{-1} K_{d} \bar{A}-I>0$. From (16), it is clear that $E$ bounded and $V(0) \geq V$. On the other hand, boundedness of $E$ implies that $E, y, \dot{y}, U_{n c}, \Phi_{y} \in \mathcal{L}_{\infty}$.

Integrating (16) gives

$$
V(t)-V(0) \leq-\int_{0}^{t} \lambda_{\min }(Q)\|E\|^{2} d \tau .
$$

Then it follows that

$$
\int_{0}^{t}\|E\|^{2} d \tau \leq \frac{V(0)}{\lambda_{\min }(Q)}<\infty .
$$

In consequence, the error $E$ is an $\mathcal{L}_{2}$ function. Boundedness of the parametric error $\widetilde{\Theta}, e$, and $\dot{e}$ in (16) allow concluding that $\dot{E}=\left[\dot{e}^{\top}, \ddot{e}^{\top}\right]^{\top}$ is an $\mathcal{L}_{\infty}$ function. The Barbalat's lemma is applied to conclude that $E$ converges to zero. Finally, from (13) it is clear that $e_{u}$ converges to zero. This completes the proof.

Parameter convergence is achieved if the following persistence of excitation condition [29]-[31] on the regressor matrix $\Phi_{y}$ is fulfilled

Definition 1: [32] A matrix $\Phi_{y}: \mathbb{R}^{n} \times \mathbb{R}^{n} \times \mathbb{R}^{n} \times \mathbb{R} \rightarrow \mathbb{R}^{p \times n}$ is persistently exciting (PE) [30] if there exist $\beta_{1}, \beta_{2}, T>0$ such that for all $t \geq 0$ the next relationship is fulfilled

$$
\beta_{1} I \leq L_{1}=\int_{t}^{t+T} \Phi_{y}(\sigma) \Phi_{y}^{\top}(\sigma) d \sigma \leq \beta_{2} I
$$

The PE condition (18) is equivalent to the uniform complete observability (UCO) [33], [34] of linear-time variant (LTV) systems.

\section{B. Estimation error}

Now consider the estimation error case, that is, the identification algorithm exhibits a small and irreducible error due to noise, disturbances or unmodelled dynamics. Consider the error dynamics (11) be rewritten as

$$
\ddot{e}=-\bar{A} \dot{e}-\bar{B} e+\Phi_{y}^{\top} \widetilde{\Theta}+\varepsilon
$$

where $\varepsilon \in \mathbb{R}^{n}$ is a bounded estimation error with $\|\varepsilon\| \leq \bar{\varepsilon}$.

The following theorem establishes that the closed-loop trajectories (19) are uniformly ultimately bounded (UUB) [17] and the parameter estimates $\widehat{\Theta}$ remain bounded under $\mathrm{PE}$ conditions.

Theorem 2: Consider the error dynamics (19). The parameters $\widehat{\Theta}$ are updated by (14) and the regressor matrix $\Phi_{y}$ fulfills the PE condition (18). Assume that there exists constants $k_{1}$ and $k_{2}$ that verifies

$$
\begin{aligned}
& k_{1}=\min \left\{\lambda_{\min }\left(K_{d} \bar{B}\right), \lambda_{\min }\left(K_{d}\left(K_{p}^{-1} K_{d} \bar{A}-I\right)\right)\right\} \\
& k_{2}=\max \left\{\lambda_{\max }\left(K_{d}\right), \lambda_{\max }\left(K_{d} K_{p}^{-1} K_{d}\right)\right\} \\
& k_{1}>\sqrt{2} k_{2} \bar{\varepsilon}+\rho
\end{aligned}
$$

where $\rho \in \mathbb{R}^{+}$. Then the trajectories of (19) are UUB and converges to a compact set $S_{E}$ of radius $\mu_{1}=\frac{\sqrt{2} k_{2} \bar{\varepsilon}}{k_{1}}$ as $t \rightarrow$ $\infty$, and therefore the parameter estimates $\widehat{\Theta}$ remain bounded. 
Proof: Consider the previous Lyapunov function (15). Its time derivative along the error dynamics (19) is

$$
\begin{aligned}
\dot{V}= & e_{u}^{\top} K_{p}^{-1}\left(K_{p} \dot{e}+K_{d} \ddot{e}\right)+\widetilde{\Theta}^{\top} \Gamma^{-1} \dot{\widetilde{\Theta}} \\
& +e^{\top}\left(K_{d} \bar{A}+\bar{B}^{\top} K_{d} K_{p}^{-1} K_{d}-K_{p}\right) \dot{e} \\
= & -e^{\top} K_{d} \bar{B} e-\dot{e}^{\top} K_{d}\left(K_{p}^{-1} K_{d} \bar{A}-I\right) \dot{e} \\
& +\widetilde{\Theta}^{\top}\left(\Gamma^{-1} \dot{\widetilde{\Theta}}+\Phi_{y} K_{d} K_{p}^{-1} e_{u}\right)+e_{u}^{\top} K_{p}^{-1} K_{d} \varepsilon
\end{aligned}
$$

If the update law is chosen as (14), then $\dot{V}$ is reduced to

$$
\begin{aligned}
\dot{V}= & -e^{\top} K_{d} \bar{B} e-\dot{e}^{\top} K_{d}\left(K_{p}^{-1} K_{d} \bar{A}-I\right) \dot{e}+e_{u}^{\top} K_{p}^{-1} K_{d} \varepsilon \\
= & -e^{\top} K_{d} \bar{B} e-\dot{e}^{\top} K_{d} \bar{K} \dot{e}+e^{\top} K_{d} \varepsilon+\dot{e}^{\top} K_{d} K_{p}^{-1} K_{d} \varepsilon \\
\leq & -\lambda_{\min }\left(K_{d} \bar{B}\right)\|e\|^{2}-\lambda_{\min }\left(K_{d} \bar{K}\right)\|\dot{e}\|^{2} \\
& +\bar{\varepsilon} \lambda_{\max }\left(K_{d}\right)\|e\|+\bar{\varepsilon} \lambda_{\max }\left(K_{d} K_{p}^{-1} K_{d}\right)\|\dot{e}\| \\
\leq & -k_{1}\|\zeta\|^{2}+\sqrt{2} k_{2} \bar{\varepsilon}\|\zeta\| \\
= & -k_{1}\|\zeta\|\left(\|\zeta\|-\frac{\sqrt{2} k_{2} \bar{\varepsilon}}{k_{1}}\right)
\end{aligned}
$$

where $\zeta=[\|e\|,\|\dot{e}\|]^{\top}$ and $\|\zeta\|=\|E\| . \dot{V}$ is negative definite if

$$
\|E\|>\frac{\sqrt{2} k_{2} \bar{\varepsilon}}{k_{1}} \equiv \mu_{1} .
$$

If $k_{1}$ satisfies (20), then the trajectories of the error dynamics (19) converge to a compact set $S_{E}$ of radius $\mu_{1}$, that is, $\|E\| \leq \mu_{1}$ and therefore, the trajectories of (19) are UUB.

From Theorem 1 is easy to show that if $E \in \mathcal{L}_{\infty}$ then the signals are bounded too, that is, $e_{u}, y, \dot{y}, \Phi_{y}, \dot{E} \in \mathcal{L}_{\infty}$. In consequence, the dynamic parametrization is bounded, that is,

$$
z \equiv \Phi_{y}^{\top} \widetilde{\Theta}=\ddot{e}+\bar{A} \dot{e}+\bar{B} e-\varepsilon
$$

which can be equivalently written as the next LTV system

$$
\begin{aligned}
& \dot{\tilde{\Theta}}=-\Gamma \Phi_{y} K_{d} K_{p}^{-1}\left[\begin{array}{ll}
K_{p} & K_{d}
\end{array}\right] E \\
& z=\Phi_{y}^{\top} \widetilde{\Theta}
\end{aligned}
$$

Since $z, E$ and $\Phi_{y}$ are bounded and $\Phi_{y}$ is PE, then the UCO condition [33] is satisfied, which guarantees boundedness of the parametric error $\widetilde{\Theta}$, and hence $\widetilde{\Theta}$. This completes the proof.

\section{NUMERICAL SIMULATION STUDIES}

The performance of the CLIE identification algorithm is assessed using a 4-DOF robot model [8] actuated by DC motors endowed with a gearbox. It is well known that a robot manipulator with gearbox train satisfies the Euler-Lagrange system (3) [35]. For this model there is no compliance term $C$ and satisfies the next Euler-Lagrange system

$$
\ddot{q}=-A \dot{q}+B u-D,
$$

where $A \in \mathbb{R}^{4 \times 4}$ denote the friction/dissipative terms, $B \in$ $\mathbb{R}^{4 \times 4}$ is the control gain, $D \in \mathbb{R}^{4}$ is the disturbance vector, $u \in \mathbb{R}^{4}$ is the control input, and $q, \dot{q}, \ddot{q} \in \mathbb{R}^{4}$ are the joint position, velocity, and acceleration vectors, respectively. For this special case, $A$ and $B$ are diagonal matrices.
TABLE I

DENAVIT HARTENBERG PARAMETERS OF THE 4-DOF ROBOT

\begin{tabular}{|c|cccc|}
\hline Joint $k$ & $\vartheta_{k}$ & $d_{k}$ & $a_{k}$ & $\alpha_{k}$ \\
\hline 1 & $q_{1}$ & $l_{1}$ & 0 & $\frac{\pi}{2}$ \\
2 & $q_{2}$ & 0 & 0 & $-\frac{\pi}{2}$ \\
3 & $q_{3}$ & 0 & $l_{3}$ & $\frac{\pi}{2}$ \\
4 & $q_{4}$ & 0 & $l_{4}$ & 0 \\
\hline
\end{tabular}

The Denavit-Hartenberg parameters [36] of the robot are given in Table I.

The joint angles of the 4-DOF robot are denoted as $q_{1}$, $q_{2}, q_{3}, q_{4}$; each link are modeled as thin bars with inertia $J_{k}^{r}=\frac{1}{12} m_{k} l_{k}^{2}$, where $m_{k}$ and $l_{k}$ are the mass and length of link $k$. The robot parameters are $m_{1}=8.4 \mathrm{~kg}, m_{2}=4.9$ $\mathrm{kg}, m_{3}=m_{4}=2.7 \mathrm{~kg}, l_{1}=0.228 \mathrm{~m}, l_{3}=l_{4}=0.22 \mathrm{~m}$. Gaussian noise $\Delta q \sim \mathcal{N}\left(0, \sigma^{2}\right)$ of small magnitude and low variance is used to model position measurement noise, that is, it is assumed that the encoder measurements exhibit a linear correlation. The Gaussian noise is obtained from the random number block of Simulink with a variance of $\sigma^{2}=1 \times 10^{-6}$ and a mean $\mu=0$. Each DC motor dynamics driving the robot links has the following inertia and friction parameters: $J_{k}^{m}=1 / 50 \mathrm{kgm}^{2}$ and $R_{k}=1 / 25 \mathrm{kgm}^{2} / \mathrm{s}$. The gear ratio $r_{k}$ is $100: 1$.

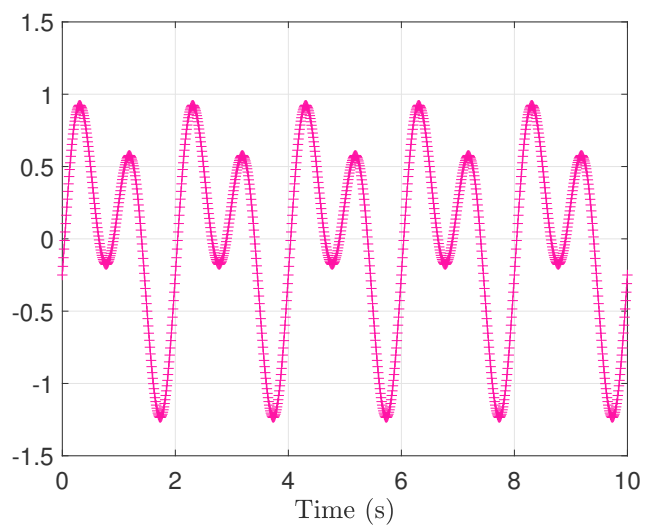

Fig. 2. PE signal

\section{A. System identification}

The regressor matrix $\Phi$ and the parameters vector $\Theta$ satisfy (5). So, there are 12 parameters to estimate. The regressor $\Phi_{y}$ and the parameter estimates vector $\widehat{\Theta}$ satisfy (9). The PE signal (see Fig. 2) is designed as a sum of sinusoidal functions with low frequency to excite the robot modes and keep tracking a smooth trajectory. The PE signal is

$$
P E=0.7 \sin (2 \pi t)+0.5 \sin (\pi t)-0.25 \cos (\pi t) .
$$

The gains of the PD controller are tuned manually until the trajectories of the robot dynamics are stabilized. The final gains are set to $K_{p}=12 I$ and $K_{d}=5 I$. The update gain is set to $\Gamma=20 I$. Fig. 3 shows the time evolution of the parameter estimates. 


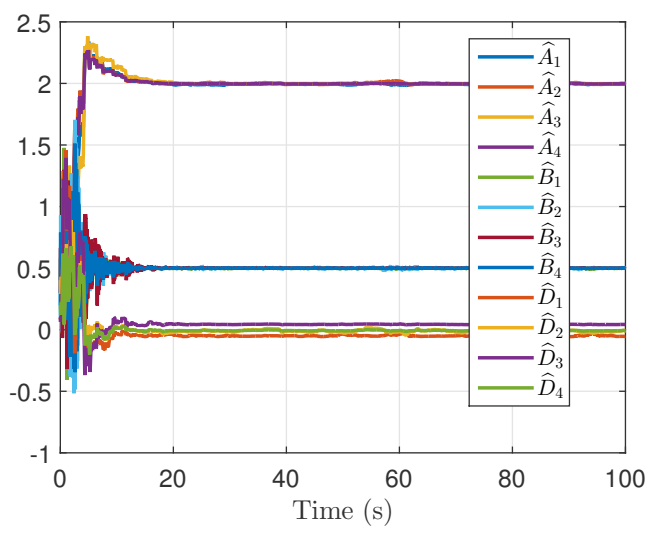

Fig. 3. Parameter estimates of the 4-DOF robot

TABLE II

PARAMETER ESTIMATES OF THE 4-DOF PLANAR ROBOT THROUGH THE CLIE UPDATE RULE (14)

\begin{tabular}{|c|c|c|c|}
\hline & Approximate & \multicolumn{2}{|c|}{ CLIE Algorithm } \\
\cline { 3 - 4 } Estimate $\widehat{\Theta}_{k}$ & Real value $\Theta_{k}$ & $\widehat{\Theta}_{k}$ & $\left|\widehat{\Theta}_{k}\right|(\boldsymbol{\%})$ \\
\hline$\widehat{A}_{1}$ & 1.9966 & 1.9963 & 0.0320 \\
$\widehat{A}_{2}$ & 1.9940 & 1.9984 & 0.4460 \\
$\widehat{A}_{3}$ & 1.9993 & 1.9999 & 0.0514 \\
$\widehat{A}_{4}$ & 1.9965 & 1.9974 & 0.0915 \\
$\widehat{B}_{1}$ & 0.4992 & 0.4987 & 0.0473 \\
$\widehat{B}_{2}$ & 0.4985 & 0.4992 & 0.0699 \\
$\widehat{B}_{3}$ & 0.4998 & 0.4998 & 0.0013 \\
$\widehat{B}_{4}$ & 0.4991 & 0.4992 & 0.0117 \\
$\widehat{D}_{1}$ & -0.0493 & -0.050 & 0.0673 \\
$\widehat{D}_{2}$ & -0.0115 & -0.0121 & 0.0610 \\
$\widehat{D}_{3}$ & 0.0431 & 0.0428 & 0.0300 \\
$\widehat{D}_{4}$ & -0.0072 & -0.0075 & 0.0266 \\
\hline
\end{tabular}

Table II summarizes the mean value of the parameters estimates and the parametric error percentage of the identification algorithm during the last 20 seconds. The results show that good estimates close to its real values are obtained.

The estimates of Table II are used to compute the estimation error $\varepsilon=\Phi_{y}^{\top} \widehat{\Theta}-\Phi^{\top} \Theta=\left[\varepsilon_{1}, \varepsilon_{2}, \varepsilon_{3}, \varepsilon_{4}\right]^{\top}$ of each identification method for 100 seconds of simulation time. The mean estimation error

$$
\bar{\varepsilon}_{k}=\frac{1}{100} \sum_{t=0}^{100} \varepsilon_{k}(t)
$$

is used to obtain the mean value of the estimation error, with $k=1 \cdots 4$. Notice that $\bar{\varepsilon}_{k}$ denote the mean estimation error of the DOF $k$. The results are: $\bar{\varepsilon}_{1}=0.4538 \times 10^{-5}, \bar{\varepsilon}_{2}=$ $-0.7173 \times 10^{-5}, \bar{\varepsilon}_{3}=0.0071 \times 10^{-5}$, and $\bar{\varepsilon}_{4}=-0.0655 \times$ $10^{-5}$. The above results verify boundedness of the estimates $\widehat{\Theta}$ and robustness again noise and nonlinear disturbances of the robot dynamics.

\section{B. Validation}

The parameter estimates of Table II are verified by computing the next feedback linearization controller

$$
u=\widehat{B}^{-1}\left[\widehat{A} \dot{q}-\widehat{D}+K_{p} \widetilde{q}+K_{d} \dot{\widetilde{q}}+\ddot{q}_{d}\right]
$$

where the same $K_{p}$ and $K_{d}$ gains of the previous identification experiment are employed; $q_{d}, \dot{q}_{d}, \ddot{q}_{d}$ are the desired joint position, velocity and acceleration, respectively. $\widetilde{q}=q_{d}-q$ and $\dot{\widetilde{q}}=\dot{q}_{d}-\dot{q}$ are the position and velocity tracking errors, respectively. The desired reference is

$$
q_{d}=\left[\begin{array}{c}
0.5 \sin \left(\frac{\pi}{6} t\right) \\
\frac{\pi}{2}+0.1 \cos \left(\frac{\pi}{6} t\right) \\
0.5 \sin \left(\frac{\pi}{6} t\right) \\
\frac{\pi}{3}+\frac{\pi}{4} \cos \left(\frac{\pi}{6} t\right)
\end{array}\right] .
$$

The forward kinematics of the robot [36] is computed using the Denavit Hartenberg parameters of Table I. The task-space trajectory is used to exhibit the accuracy of the the feedback linearization controller under the parameter estimates of Table II. The task-space trajectory results are shown in Fig. 4.

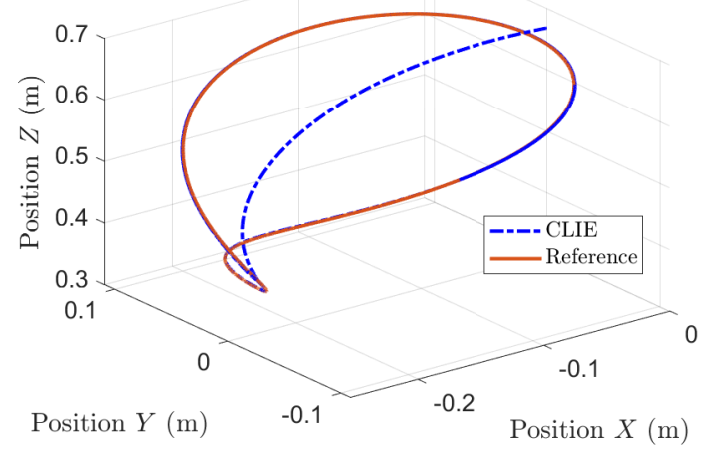

Fig. 4. Trajectory in the task space of the 4-DOF robot

The results show an accurate tracking performance of the identification method. The mean squared error

$$
\bar{q}_{k}=\frac{1}{100} \sum_{t=0}^{100} \widetilde{q}_{k}^{2}(t)
$$

of the tracking error $\widetilde{q}$ is used to show the accuracy of the feedback linearization controller under the estimates of Table II. The numerical results are: $\bar{q}_{1}=0.1322 \times 10^{-4}, \bar{q}_{2}=$ $0.0253 \times 10^{-4}, \bar{q}_{3}=0.0055 \times 10^{-4}$, and $\bar{q}_{4}=0.0059 \times 10^{-4}$. These outcomes show that the proposed approach obtains reliable estimates that are close to their real values.

\section{CONCLUSION}

This paper provides an input error identification method for parameter identification of a class of Euler-Lagrange systems. The approach uses the input error instead of the output error to update a gradient identification law. The regressor matrix uses measurements of the estimated model's states instead of the states of the real system such that noise and high-pass filter are avoided. Stability and convergence of the complete closedloop system is assessed using Lyapunov stability theory under persistency of exciting conditions. Numerical simulations are carried out to validate the proposed approach. 


\section{REFERENCES}

[1] R. Kelly and V. Santibánez, Control of Robot Manipulators in Joint space. Pearson, Prentice Hall, 2003.

[2] R. Garrido and R. Miranda, "DC servomechanism parameter identification: A closed loop input error approach," ISA Transactions, vol. 51, pp. 42-49, 2012.

[3] S. Soliman, A. Al-Kandari, and M. El-Hawari, "Parameter identification method of a separately excited DC motor for speed control," Electric Machines and Power Systems, vol. 6, pp. 615-626, 1998.

[4] T. Yamammoto, M. Bernhardt, A. Peer, M. Buss, and A. M. Okammura "Techniques for Environment parameter Estimation during telemanipulation," Proceedings of the 2nd Biennial IEEE/RAS-EMBS International Conference on Biomedical Robotics and Biomechatronics, 2008.

[5] R. Garrido and R. Miranda, "Closed loop Identification of a DC servomechanism," IEEE 10th International Conference on Power Electronics CIEP, 2006.

[6] C. Fuh and H. Tsai, "Adaptive parameter identification of servo control systems with noise and high-frequency uncertainties," Mechanical Systems and Signal Processing, vol. 21, pp. 1237-1251, 2007.

[7] J. E. Slotine and W. Li, Applied Nonlinear Control. Prentice Hall, 1991.

[8] W. Yu and A. Perrusquía, "Simplified stable admittance control using end-effector orientations," International Journal of Social Robotics, vol. 12, pp. 1061-1073, 2020.

[9] L. Sciavicco Brui, Modeling and Control of Robots Manipulators Springer, 2005.

[10] A. Perrusquía, J. A. Flores-Campos, and W. Yu, "Optimal sliding mode control for cutting tasks of quick-return mechanisms," ISA transactions, 2021.

[11] G. Song, L. Cai, Y. Wang, and R. W. Longman, "A Sliding-mode based smooth adaptive robust controller for friction compensation," International Journal of Robust and Nonlinear Control, pp. 725-739, 1998.

[12] J. A. Flores-Campos, A. Perrusquía, L. H. Hernández-Gómez, N. González, and A. Armenta-Molina, "Constant speed control of slidercrank mechanisms: A joint-task space hybrid control approach," IEEE Access, vol. 9, pp. 65676-65687, 2021.

[13] A. Perrusquía and W. Yu, "Human-in-the-loop control using euler angles," Journal of Intelligent \& Robotic Systems, vol. 97, pp. 271-285, 2020.

[14] S. Zeng and E. Fernandez, "Adaptive controller design and disturbance attenuation for sequantially interconnected SISO linear systems under noisy output measurements with partly measured disturbances," 2008 American Control Conference, 2008.

[15] A. Perrusquía and W. Yu, "Identification and optimal control of nonlinear systems using recurrent neural networks and reinforcement learning: An overview," Neurocomputing, vol. 438, pp. 145-154, 2021.

[16] A. Perrusquía, W. Yu, A. Soria, and R. Lozano, "Stable admittance control without inverse kinematics," 20th IFAC World Congress (IFAC2017), vol. 50, no. 1, pp. 15835-15840, 2017.

[17] H. K. Khalil, Nonlinear Systems. Prentice Hall, third ed., 2002.

[18] A. Perrusquía and W. Yu, "Continuous-time reinforcement learning for robust control under worst-case uncertainty," International Journal of Systems Science, vol. 52, no. 4, pp. 770-784, 2021.

[19] A. Perrusquía, W. Yu, and A. Soria, "Position/force control of robot manipulators using reinforcement learning," Industrial Robot: the international journal of robotics research and application, vol. 46, no. 2 , pp. 267-280, 2019

[20] G. De Gersem, H. Van Brussel, and J. Vander Sloten, "Enhanced haptic sensitivity for soft tissues using teleoperation with shaped impedance reflection," World Haptics Conference (WHC), 2005.

[21] R. Corteso, J. Park, and O. Khatib, "Real-time adaptive control for haptic telemanipulation with kalman active observers," IEEE Transactions on Robotics, vol. 22, no. 5, pp. 987-999, 2006.

[22] I. Landau and A. Karimi, "An output error recursive algorithm for unbiased identification in closed loop," Automatica, vol. 33, no. 5, 1997.

[23] M. Brunot, A. Janot, F. Carrillo, C. Joono, and J.-P. Noël, "Output error methods for robot identification," Journal of Dynamic Systems Measurement and Control, 2019.

[24] R. Garrido and R. Miranda, "Autotuning of a de servomechanism using closed loop identification," 32nd Annual Conference on IEEE Industrial Electronics (IECON), 2006.
[25] M. Gautier, A. Janot, and P.-O. Vandanjon, "A new closed-loop output error method for parameter identification of robot dynamics," IEEE Transactions on Control Systems Technology, vol. 21, no. 2, 2013.

[26] M. Gautier and W. Khalil, "Exciting trajectories for the identification of base inertial parameters of robots," In Proceedings of the 30th IEEE Conference on Decision and Control, 1991.

[27] A. Perrusquía, J. A. Flores-Campos, and C. R. Torres San-Miguel, "A novel tuning method of PD gravity compensation controller for robot manipulators," IEEE Access, vol. 8, pp. 114773-114783, 2020.

[28] A. Perrusquía, J. A. Flores-Campos, C. R. Torres-Sanmiguel, and N. González, "Task space position control of slider-crank mechanisms using simple tuning techniques without linearization methods," IEEE Access, vol. 8, pp. 58435-58442, 2020.

[29] S. Sastry and M. Bodson, Adaptive Control, Stability, Convergence and Robustness. Prentice Hall, 1989.

[30] C. Canudas de Wit and A. Aubin, "Robot parameter identification via sequential hybrid estimation algorithm," European Control Conference, pp. 444-449, 1991.

[31] A. Perrusquía and W. Yu, "Discrete-time $\mathcal{H}_{2}$ neural control using reinforcement learning," IEEE Transactions on Neural Networks and Learning Systems, 2020.

[32] A. Perrusquía, W. Yu, and X. Li, "Nonlinear control using human behavior learning," Information Sciences, vol. 569, pp. 358-375, 2021.

[33] F. Lewis, S. Jagannathan, and Yeşildirek, Neural Network control of robot manipulators and nonlinear systems. Taylor \& Francis, 1999.

[34] A. Perrusquía and W. Yu, "Neural $\mathcal{H}_{2}$ control using continuous-time reinforcement learning," IEEE Transactions on Cybernetics, 2020.

[35] M. W. Spong and M. Vidyasagar, Robot dynamics and control. John Wiley \& Sons, 2008.

[36] A. Perrusquia, W. Yu, and X. Li, "Multi-agent reinforcement learning for redundant robot control in task-space," International Journal of Machine Learning and Cybernetics, vol. 12, no. 1, pp. 231-241, 2021. 
$2021-12-14$

\section{An input error method for parameter identification of a class of \\ Euler-Lagrange systems}

Perrusquía, Adolfo

IEEE

Perrusquía A, Garrido R, Yu W. (2021) An input error method for parameter identification of a class of Euler-Lagrange systems. In: 18th International Conference on Electrical Engineering, Computing Science and Automatic Control (CCE), 10 November - 12 October 2021, Mexico City, Mexico

https://doi.org/10.1109/CCE53527.2021.9633110

Downloaded from Cranfield Library Services E-Repository 